NASA Technical Memorandum 109095

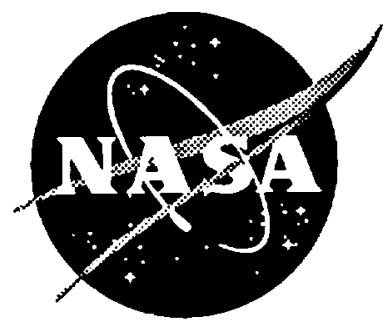

\title{
Preliminary Evaluation of Hybrid Titanium Composite Laminates
}

J. L. Miller, D. J. Progar, W. S. Johnson, and T. L. St. Clair Langley Research Center, Hampton, Virginia

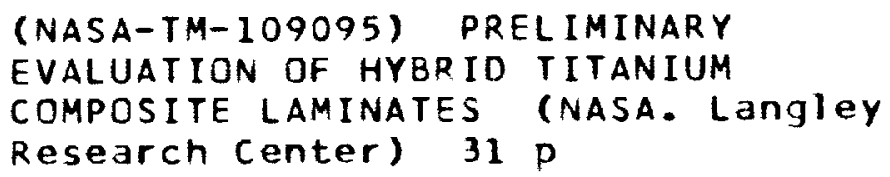

National Aeronautics and Space Administration Langley Research Center Hampton, Virginia 23681-0001 


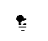




\title{
PRELIMINARY EVALUATION OF HYBRID TITANIUM COMPOSITE LAMINATES
}

\author{
J. L. Miller ${ }^{1}$, D. J. Progar ${ }^{2}$, W. S. Johnson ${ }^{3}$ and T. L. St. Clair ${ }^{2}$
}

\begin{abstract}
In this study, the mechanical response of hybrid titanium composite laminates (HTCL) was evaluated at room and elevated temperatures. Also, the use of an elastic-plastic laminate analysis program for predicting the tensile response from constituent properties was verified. The improvement in mechanical properties achieved by the laminates was assessed by comparing the results of static strength and constant amplitude fatigue tests to those for monolithic titanium sheet. Two HTCL were fabricated with different fiber volume fractions, resin layer thicknesses and resins. One panel was thicker and was poorly bonded in comparison to other. Consequently, the former had a lower tensile strength, while fewer cracks grew in this panel and at a slower rate. Both panels showed an improvement in fatigue life of almost two orders of magnitude. The model predictions were also in good agreement with the experimental results for both HTCL panels.
\end{abstract}

Keywords: adhesive bonding, graphite fibers, isothermal fatigue, laminated titanium, crack growth.

\section{INTRODUCTION}

Both military and commercial aircraft are being designed to fly faster and to last longer than ever before. This requires structural materials that are capable of operating at higher temperatures yet exhibit improved fatigue and damage tolerance properties. One material system that merits attention is a hybrid titanium and polymeric matrix composite laminate.

$\overline{1}$ National Research Council Resident Research Associate

${ }^{2}$ Senior Scientist

${ }^{3}$ Senior Research Engineer 
In the mid 1960's Kaufman [1] showed that the fracture toughness of adhesively laminated aluminum plies was improved in comparison to that of an equivalent monolithic plate due to the individual plies failing in a plane stress state. In the mid 70's Johnson and colleagues $[2,3]$ showed that significant improvements in fatigue and crack growth resistance could also be realized by adhesively laminating thin aluminum plies together. In the early 80 's Johnson extended this work to show that adhesively laminated titanium plies improved fracture toughness by almost $40 \%$, increased fatigue life by an order of magnitude, and slowed down though-thethickness crack growth rates by $20 \%$ [4]. In the mid 80 's researchers at Delft University and Alcoa developed ARALL (Aramid Reinforced Aluminum Laminates) where not only were thin plies of aluminum adhesively laminated together, but aramid fibers were also included in the bondline $[5,6]$. The inclusion of the fibers offered even more improvement in mechanical behavior of the laminate, primarily due to reductions in crack growth rates resulting from a fiber bridging phenomenon. Some of the ARALL laminates were even prestrained so the aluminum plies were in a state of residual compression, further improving fatigue properties. Similar improvements have been offered by GLARE laminates [7] that are essentially the same concept, but glass fibers replace the aramid. Thus, the history of laminated metals has shown definite mechanical advantages that can translate to weight savings. These laminated aluminum parts are now flying on several commercial and military aircraft.

This preliminary study will build upon past experience and apply the laminated/hybrid technology to higher temperature metals and adhesives in hopes of demonstrating that these systems will be useful in high speed aircraft. Two unidirectional hybrid titanium composite laminates (HTCL) were made with different fiber volume fractions, resin layer thicknesses and resins. From the limited supply of material, the HTCL were tested statically to failure and cyclically in fatigue. The results were compared to each other and to an similar thickness piece of monolithic titanium to assess lamination affects. A laminate analysis code was used to predict the hybrid laminate response based upon constituent property input and to verify is applicability to model the mechanical response of this type of material system. 


\section{MATERIALS}

The HTCL were fabricated as $7.6 \mathrm{~cm} \times 17.8 \mathrm{~cm}$ panels with Ti-6Al-4V alloy for the metal portion of the laminated panel. Prior to laminating, the titanium alloy was given the following Pasa-Jell $107^{*}$ surface treatment:

1. Washed with acetone

2. (Panel 1) Abraded surface with 150 grit sandpaper

(Panel 2) Gritblasted with 120 aluminum oxide grit

3. Washed with methyl alcohol

4. Brushed Pasa-Jell 107 on surfaces, let set for $10 \mathrm{~min}$

5. Repeat 4.

6. Washed with hot running tap water, then cold tap water

7. Placed in ultrasonic dimineralized water bath for $15 \mathrm{~min}$

8. Removed and air dried in laboratory hood for $10 \mathrm{~min}$

9. Dried in forced-air oven at $100^{\circ} \mathrm{C}$ for $10 \mathrm{~min}$

10. Primed within two hours of treatment

Two HTCL were prepared using two different polyimide adhesive materials. The prepreg (adhesive tape) was made in-house (Polymeric Materials Branch) on a multipurpose prepregging machine.

Panel 1 was prepared using LARC ${ }^{\text {TM }}$-IA 4 mole $\%$ offset $[8$ mole $\%$ phthalic anhydride (PA) end cap] in N-methyl-2-pyrrolidone (NMP) [8] and IM7 graphite fibers as a unidirectional powder coated prepreg, approximately $0.33 \mathrm{~mm}$ thick. The LARC ${ }^{\text {TM}}-I A$ was obtained from Imitec. The primer, which was prepared in-house, was applied to the titanium as a $10.6 \mathrm{wt}$. \% LARC $^{\text {TM }}$-IA 3 mole \% offset ( 6 mole \% PA) amic acid solution in gamma-butyrolactone. The solution was brushed on the titanium and staged in a forced-air oven for $15 \mathrm{~min}$ each at $50^{\circ}, 100^{\circ}$ and $225^{\circ} \mathrm{C}$.

Panel 2 was prepared with LARC ${ }^{\text {TM }}$-IAX [a version of LARC ${ }^{\text {TM }}$-IA in which the backbone was modified slightly with 10 mole $\%$ of a more rigid diamine, p-phenylenediamine, to improve solvent resistance] amic acid (again a 4 mole \% offset as with LARC ${ }^{\mathrm{TM}}-\mathrm{IA}$ ), $30 \mathrm{wt}$ \% solids solution in NMP with a viscosity of $30,400 \mathrm{cp}$ and IM7 fibers solution coated as a unidirectional prepreg approximately $0.18 \mathrm{~mm}$ thick. The titanium sheets were primed with a 7.5

*Trade name for titanium surface treatment available from Semco, Glendale, CA, USA 
wt \% solution of LARC ${ }^{\text {TM }}$-IAX in NMP and air dried for one hour after which they were placed in a forced-air oven and heated for one hour each at $150^{\circ}$ and $230^{\circ} \mathrm{C}$.

\section{MANUFACTURING and PROCESSING}

The laminate assembly was arranged in an open-ended matched-die mold by alternating six layers of titanium sheets with five layers of adhesive tape. TX1040 release cloth was placed on the bottom and top of the laminate assembly to prevent the laminate from sticking to the mold. The mold had previously been coated with a mold release agent. A hydraulic press with $30.5 \mathrm{~cm} \times 30.5 \mathrm{~cm}$ heated platens and load cell was used to process the laminates.

The initial attempt to process Panel 1 , under $0.10 \mathrm{MPa}$ at $343^{\circ} \mathrm{C}$ for one hour, did not appear to provide adequate flow in the adhesive, as indicated by the lack of any excess adhesive polymer outside the laminate and the thickness of the laminate. Therefore, the laminate was further processed at a pressure of $13.8 \mathrm{MPa}$ at $371^{\circ} \mathrm{C}$ for one hour. The average adhesive thickness per layer was determined to be $0.28 \mathrm{~mm}$.

Panel 2 was processed slightly differently than Panel 1. The laminate assembly was arranged in the bottom part of the die mold, placed in a forced-air oven without the top part of the die mold, then staged to remove some of the volatiles prior to the bonding procedure. The laminate assembly was heated for one hour each at $150^{\circ}, 175^{\circ}$ and $230^{\circ} \mathrm{C}$. The laminate assembly with the top part of the die mold was then placed in the press where a pressure of 13.8 $\mathrm{MPa}$ was applied while heating the assembly to $350^{\circ} \mathrm{C}$ in approximately one hour. The pressure and temperature was then held for one hour. The average adhesive thickness for Panel 2 was $0.12 \mathrm{~mm}$. Schematic diagrams indicating the thickness variations are shown in Figure 1.

\section{ANALYTICAL METHODS}

The AGLPLY code [9], developed originally to analyze metal matrix composites, was used to evaluate its potential for predicting the HTCL's response based on constituent input. The

The use of trade names in this paper does not constitute endorsement, either expressed or implied, by the National Aeronautics and Space Administration. 
program performs an elastic-plastic analysis of symmetric composite laminated plates under inplane mechanical loads. The lamina properties are calculated via the vanishing fiber diameter (VFD) model which assumes a rule of mixtures contribution of the fiber to the modulus in the longitudinal direction while offering no transverse constraint by the fiber [9]. AGLPLY computes the overall laminate elastic moduli, the local fiber and matrix stresses and strains in each ply as well as the overall laminate strains for the entire elastic-plastic loading regime. This program has proven successful in predicting the mechanical response of metal matrix composites in numerous studies [10-12]. Table 1 displays the constituent properties used to predict the composite properties.

\section{EXPERIMENTAL PROCEDURE}

Tests were conducted on both monolithic Ti-6Al-4V and the two HTCL. From the limited supply of material manufactured, six HTCL specimens were fabricated, three from each panel. One specimen from each panel was tested statically to failure to examine the tensile behavior and two were tested cyclically in fatigue, one at room temperature and the other at $177^{\circ}$ C. To assure a valid comparison between the monolithic material and the titanium in the HTCL, Rockwell A hardness tests (RHA) were conducted on both materials. Since the lamination process involves elevated temperatures, it was of interest to determine whether the titanium was affected by this processing. The titanium laminae and the monolithic sheet were both 68 RHA; thus it is assumed that the titanium laminae are the same as the monolithic material, only varying in thickness.

The fiber volume fraction in the laminates were determined by digital image analysis of polished ( $2.5 \mu \mathrm{m}$ finish) cross sections using an image processing system. A series of digital image scans were made over the adhesive layers and the average volume fraction of fibers was determined from the area fraction of the fibers measured in each scan. Twenty scans were made for Panel 1 and ten scans were made for Panel 2. Greater variations in fiber distribution in Panel 1 necessitated more scans. 
The room temperature tensile response was evaluated using $19 \mathrm{~mm}$ wide straight-sided specimens. The specimens were tested to failure in laboratory air at a loading rate of $150 \mathrm{~N} / \mathrm{sec}$ on a $250 \mathrm{kN}$ servo-hydraulic test frame, equipped with hydraulic grips; the applied gripping pressure was $14 \mathrm{MPa}$. Axial strain was measured on the surface of the specimen using a strain gage extensometer with a $25 \mathrm{~mm}$ gage section. Results were recorded on an X-Y plotter.

Constant amplitude fatigue tests were conducted on straight-sided specimens, $19 \mathrm{~mm}$ wide (W), containing a $6.4 \mathrm{~mm}$ diameter (d) center hole, rending $\mathrm{d} / \mathrm{W}=0.33$. The holes in the monolithic titanium were produced by electronic discharge machining, while the HTCL were drilled. The interior of the holes in both materials had the same smooth surface finish. The surface of the specimens were polished along the gage section to a $3 \mu \mathrm{m}$ finish. All tests were conducted on a $250 \mathrm{kN}$ servo-hydraulic test frame at a cyclic frequency of $10 \mathrm{~Hz}$ and an $\mathrm{R}=0.1$. The HTCL were evaluated for their fatigue resistance by applying equivalent load-to-weightratios as compared to the monolithic material. The densities of the materials were $2.9,3.4$ and $4.4 \mathrm{~g} / \mathrm{cc}$ for Panel 1, Panel 2 and the monolithic sheet, respectively. Variations in the thickness of the adhesive layers resulted in differing laminate densities. The resulting applied loads for the laminates were 0.67 and 0.78 times the applied load in the monolithic material. Elevated temperature fatigue tests were performed at $177^{\circ} \mathrm{C}$ in a convection oven mounted on the test stand. A K-type thermocouple positioned on the surface of the specimen, away from the hole, indicated the test temperature.

Crack growth measurements were made during the room temperature testing by replicating the cracked region near the hole with an acetate film. A mirror image of the crack is created in the film which can then be examined microspcopically and measured. Both the front and back surfaces of the specimen were replicated. This technique was not applicable for the elevated temperature tests. During these tests, crack lengths were measured with a long focal length microscope and video imaging system by viewing the specimen surface through a window in the oven. Thus, crack measurements were made on the front surface only for the elevated temperature tests. 


\section{RESULTS AND DISCUSSION}

Average fiber volume fractions were determined for each panel. Panel 1, the thicker laminate, had $44.0 \pm 8.6 \%$ fibers while Panel 2 had $63.8 \pm 5.0 \%$ fibers in the adhesive layers. Optical micrographs of the polished cross sections (Figure 2) show significant variations in the fiber distribution in the two laminates: the fibers were much more uniformly distributed throughout the adhesives layers in Panel 2, whereas many resin rich areas existed in Panel 1. This non-uniformity contributes to the larger variation in the measured average volume fraction for Panel 1.

The results of room temperature tension tests on the monolithic titanium and the HTCL are shown in Figure 3. Due to the limited supply of HTCL, elevated temperature tensile response was not evaluated. Panel 1 performed poorly in tension, failing at a lower strain than the monolithic titanium and showing no improvement in elastic modulus. Panel 2 showed a significant improvement in tensile strength, approximately doubling the strength of the titanium alone, as well as an improved elastic modulus. The fracture modes of the two HTCL varied greatly as well (Figure 4). Panel 1 suffered severe delamination during the test, a result of the poor bonding between the adhesive and the titanium layers. In Panel 2, the titanium plies showed more plastic deformation and the amount of delamination was less. In Panel 1 the delaminations and fractured plies extended throughout the gage length of the specimen, while in Panel 2, the damage was more localized.

The elastic-plastic laminate analysis model AGLPLY was used to predict the stress-strain response of the various materials used in this study. Prior to applying the model to the HTCL, the applicability of the model to predict behavior of the individual lamina was verified. Figure 5 displays the model predictions bounding the experimental data [13] for the tensile response of the IM7/LARC ${ }^{\text {TM }}$-IA polymer composite over the applicable range of fiber volume fractions for the composites tested. For the HTCL the predictions over the range of measured fiber volume fractions are compared directly with the experimental data for Panels 1 and 2, in Figures 6 and 7 , respectively. Overall the predictions were in good agreement with the experimental results. For 
both panels, the prediction using the lowest fiber volume fraction gives the best representation of the experimental results in each case: both the elastic modulus and the tensile strength are in excellent agreement while yielding in the titanium plies occurs as indicated by the change in slope, at approximately 820 and $900 \mathrm{MPa}$ laminate stress for Panel 1 and Panel 2, respectively. Figure 8 compares the experimental results to the AGLPLY predictions for the monolithic titanium and the HTCL. The shape of the prediction curves closely resemble the experimental results, with the change in slope representing the yielding that occurs when the stress in the titanium reaches $820 \mathrm{MPa}$. The predictions also predict the elastic moduli fairly well as shown in Figure 8.

Results of the constant amplitude fatigue tests on center hole specimens of the monolithic titanium and the HTCL are shown in Figure 9. For the monolithic titanium, increasing the temperature reduces the overall fatigue life of the titanium. For the HTCL, all the specimens tested were able to withstand $10^{6}$ cycles without fracturing the laminate completely, both at room and elevated temperature. However, the panels were tested at an equivalent load-to weight ratio which results in different applied stresses for the individual panels due to differences in thickness and density. Figure 10 shows the results in terms of the equivalent load-to-weight ratio tested. The HTCL panels showed a marked improvement in fatigue life of almost two orders of magnitude at both room and elevated temperatures. Further comparing the results on the basis of equivalent stress-to-weight ratios, as shown in Figure 11, illustrates the benefit of the lower density of the HTCL: the thinner, lighter weight Panel 2, has a greater stress per unit weight than the monolithic titanium, yet has a longer fatigue life. Similar results are shown for Panel 1.

Crack propagation was monitored on all HTCL specimens. Cracks initiated at the hole, growing through the exterior titanium plies and then linking with the interior plies. Figure 12 shows the edge replicas of a specimen from Panel 2, taken at different stages during the test, illustrating this pattern. Cracks had propagated through all the titanium plies at 890,000 cycles, yet the specimen remained whole and continued to carry load until the test was stopped at a million cycles. Once the titanium was completely cracked, the fibers carried all the load. The 
stress in the fibers (based on the average volume fraction) with only the fibers carrying load, was calculated to be $445 \mathrm{MPa}$ for Panel 1 and $495 \mathrm{MPa}$ for Panel 2. These fiber stresses are much less than the fiber ultimate strength $(5310 \mathrm{MPa})$, and therefore, the fibers would continue to carry the applied load indefinitely without fracturing. If the same loads were applied to the monolithic titanium, failure would be expected since these stresses are greater than the endurance limit of the alloy (138 MPa) as shown in Figure 9.

Typically in metallic materials, the crack propagation rate increases with crack growth exponentially. For laminated sheets, the crack growth is faster initially, but slows as the crack encounters the interfaces between layers, yielding an overall longer fatigue life, as shown by Johnson[4]. However, for the HTCL tested in this study, the crack growth rate remains constant, as indicated by the linear curves in Figures 13-15. Since crack growth was not monitored in the monolithic titanium, predictions were made using FASTRAN-II, a fatigue crack growth structural analysis program [14]. This program has been used extensively to predict fatigue crack growth from available crack growth data on specific alloys. An assumed initial crack size of $1 \mathrm{~mm}$ was used for these predictions to illustrate the extent of the reduction in the crack growth rate in the HTCL as compared to monolithic titanium. These results are also shown in Figures 13 and 14. The predictions indicate an increasing rate rather than the constant rate of growth shown by the HTCL. The reduced crack growth in the HTCL is attributed to fiber bridging, where the fibers in the adhesive layers act to bridge the gap across the cracks in the titanium. The result is a reduced stress intensity at the crack tip and slower crack growth in the HTCL than in the monolithic material. The phenomena of fiber bridging is active in composite material systems [15-16] and contributes to the improved damage tolerance of these materials.

Cracks propagated significantly faster in Panel 2 than in Panel 1, at room and at elevated temperature. Cracks propagated at much slower rates in both HTCL than predicted for the monolithic material. Crack growth rates for the two laminates were calculated for each individual crack, as shown in Table 2. In the monolithic titanium, the predicted crack growth rate varies greatly depending on the length of the crack. For the monolithic titanium at an 
applied stress $130 \mathrm{MPa}$ (same as in Panel 1), to extend a $1 \mathrm{~mm}$ crack to $1.5 \mathrm{~mm}$, a $3 \mathrm{~mm}$ to 3.5 $\mathrm{mm}$, and a $5 \mathrm{~mm}$ to $5.5 \mathrm{~mm}$, the crack growth rates would be $9.72 \mathrm{E}-5 \mathrm{~mm} / \mathrm{cycle}, 3.29 \mathrm{E}-4$ $\mathrm{mm} /$ cycle and $3.91 \mathrm{E}-3 \mathrm{~mm} /$ cycle, respectively. In Panel 1, the highest rate is $1.67 \mathrm{E}-5 \mathrm{~mm} / \mathrm{cycle}$, over 200 times slower than the growth in the monolithic titanium at a crack length of $5 \mathrm{~mm}$. Similarly, for monolithic material at an applied stress $211 \mathrm{MPa}$ (same as in Panel 2), to extend a crack from $4.5 \mathrm{~mm}$ to $5 \mathrm{~mm}$ the average crack growth rate would be $1.62 \mathrm{E}-2 \mathrm{~mm} / \mathrm{cycle}$, whereas in Panel 2, the highest crack growth rate was $9.80 \mathrm{E}-5 \mathrm{~mm} / \mathrm{cycle}$, over 150 times slower. In addition, the predictions indicate that in the monolithic material crack growth becomes unstable and rapid fracture ensues, at crack lengths greater than $5 \mathrm{~mm}$.

The poor bonding of Panel 1 may have reduced the amount of load transfer from the fibers in the adhesive plies to the titanium plies. Therefore, a weaker bond may allow the fibers to be the dominant load carrying component. In fatigue, a weak bond is an advantage, but at compromise is made in ultimate strength, as is evident by the behavior of Panel 1 (weak bond) and Panel 2(strong bond).

\section{CONCLUSIONS}

This investigation evaluated the tensile response and the fatigue resistance of HTCL at room temperature. The effect of elevated temperature on fatigue resistance was also examined. The laminate analysis code AGLPLY was used to predict the HTCL response based upon constituent property input. Results of these tests were compared to those for monolithic titanium sheets to assess the improvement in mechanical behavior attained by the HTCL. Two laminates were fabricated from different resins and of differing fiber volume fractions and resin layer thicknesses, then tested statically in tension and under constant amplitude fatigue loading. The crack growth was monitored and recorded via acetate film replication.

The experiments showed that the quality of the adhesive bond greatly influences the mechanical properties achievable through lamination. A strong bond allows load transfer between the plies without delamination occurring, producing a higher strength material, as in the 
case of Panel 2. However, if fatigue resistance is the major concern, a weaker bond, as in Panel 1 , reduces the amount of load transfer, allowing the fiber strength to dominate. Both HTCL have longer fatigue lives than the monolithic titanium, lasting over a million cycles at both room and elevated temperatures, at applied stresses of $130 \mathrm{MPa}$ and $211 \mathrm{MPa}$ for Panel 1 and Panel 2, respectively. Cracking in the titanium plies did occur, but the specimens did not fracture. Fiber bridging was assumed to dominate the crack growth process. Once all the titanium plies were fractured, the fibers continued to carry the applied load. The elastic-plastic laminate analysis model AGLPLY was shown to predict the laminates' tensile response fairly accurately, verifying its applicability to modeling the mechanical response of the HTCL. Overall, the HTCL provide a stronger, stiffer and more damage tolerant alternative for higher temperatures to monolithic materials while adding a reduction in weight, properties necessary for applications to future high speed aircraft.

\section{REFERENCES}

1. Kaufman, J. G., "Fracture Toughness of 7075-T6 and -T651 Sheet, Plate and Multilayered Adhesive-Bonded Panels," Journal of Basic Engineering, Vol. 89, 1967, pp. 503-507.

2. Johnson, W. S. and Stratton, J. M., "Effective Remote Stresses and Stress Intensity Factors for an Adhesive Bonded Multi-Ply Laminate," Engineering Fracture Mechanics, Vol. 9, 1977, pp. 411-421.

3. Johnson, W. S., Rister, W. C. and Spamer, T., "Spectrum Crack Growth in Adhesively Bonded Structure," Journal of Engineering Materials and Technology, Vol. 100, 1978, pp. 57-63.

4. Johnson, W. S., "Damage Tolerance Evaluation of Adhesively Laminated Titanium," Journal of Engineering Materials and Technology, Vol. 105, 1983, pp. 182-187.

5. Vogelesang, L. B. and Gunnink, J. W., "ARALL, A Material for the Next Generation of Aircraft. A State of the Art," Report LR-400, Dept. of Aerospace Engineering, Delft University of Technology, The Netherlands (1983).

6. Bucci, R. J., Mueller, L. N., Schultz, R. W., and Prohaska, J. L., "ARALL-Laminates-Results from a Cooperative Test Program," Advanced Materials Technology '87, Society for the Advancement of Material and Process Engineering, Vol. 32, 1987, pp. 902-916.

7. Schijve, J., "Development of Fibre-Metal Laminates, ARALL and GLARE, New Fatigue Resistant Materials," FATIGUE '93, May 3-7, 1993, pp. 3-20.

8. Progar, D. J. and St. Clair, T. L., "A New Flexible Backbone Polyimide Adhesive," Journal of Adhesion Science and Technology, Vol. 4, No. 7, 1990, pp. 527-549. 
9. Bahei-El-Din, Y. A. and Dvorak, G. J., "Plasticity Analysis of Laminated Composite Plates," Journal of Applied Mechanics, Vol. 49, 1982, pp. 740-746.

10. Johnson, W. S., "Modeling Stiffness Loss in Boron/Aluminum Laminates Below the Fatigue Limit," Long Term Behavior of Composites, ASTM STP 813, T. K. O'Brien, Ed., American Society of Testing and Materials, Philadelphia, 1983, pp. 160-176.

11. Johnson, W. S., "Fatigue Behavior of Continuous-Fiber Silicon Carbide/Aluminum Composites," Composite Materials: Fatigue and Fracture, ASTM STP 907, H. T. Hahn, Ed., American Society for Testing and Materials, Philadelphia, 1986, pp. 161-175.

12. Johnson, W. S., Lubowinski, S. J. and Highsmith, A. L., "Mechanical Characterization of Unnotched SCS-6/Ti-15-3 Metal Matrix Composites at Room Temperature," Thermal and Mechanical Behavior of Metal Matrix and Ceramic Matrix Composites, ASTM STP 1080, J. M. Kennedy, H. H. Moeller, and W. S. Johnson, Eds., American Society for Testing and Materials, Philadelphia, 1990, pp. 193-218.

13. Hou, T. H., Johnston, N. J. and St. Clair, T. L., "Processing and Properties of IM7/LARC ${ }^{\mathrm{TM}}$-IA Polyimide Composites," to be published in the Proceedings of the 39th International SAMPE Symposium and Exhibition, Anaheim, CA, April 11-14, 1994.

14. Newman, J. C., Jr. "FASTRAN-II--A Fatigue Crack Growth Structural Analysis Program," NASA Technical Memorandum 104159, February 1992.

15. Shaw, M. C., Marshall, D. B. and Evans, A. G., "Bridging Process in Metal-Reinforced Ceramics," Materials Research Society Symposium Proceedings, Vol. 170, 1990, pp. 25-31.

16. Bakuckas, J. G., Jr. and Johnson, W. S., "Application of Fiber Bridging Models to Fatigue Crack Growth in Unidirectional Titanium Matrix Composites," Journal of Composites Technology and Research, Vol. 15, No. 3, 1993, pp. 242-255. 
Table 1. Constituent material properties of titanium hybrid composite.

\begin{tabular}{|c|c|c|c|c|c|c|}
\hline \multirow{3}{*}{$\begin{array}{l}\text { Yield Strength } \\
\text { Ultimate Strength }\end{array}$} & \multicolumn{2}{|c|}{$\mathrm{Ti}-6 \mathrm{Al}-4 \mathrm{~V}^{\mathrm{a}}$} & \multicolumn{2}{|c|}{ LARC $^{\mathrm{TM}}-\mathrm{IA}^{\mathrm{b}}$} & \multicolumn{2}{|c|}{ IM7 } \\
\hline & 827.4 & $\mathrm{MPa}$ & 71.7 & $\mathrm{MPa}$ & \multicolumn{2}{|c|}{ N/A } \\
\hline & 1068.7 & $\mathrm{MPa}$ & 121.9 & $\mathrm{MPa}$ & 5310.0 & $\mathrm{MPa}^{\mathrm{c}}$ \\
\hline Longitudinal Modulus & 118.6 & $\mathrm{GPa}$ & 3.34 & GPa & 275.8 & $\mathrm{GPa}$ \\
\hline Transverse Modulus & & & & & 13.79 & $\mathrm{GPa}$ \\
\hline Shear Modulus & 45.62 & $\mathrm{GPa}$ & 1.26 & $\mathrm{GPa}$ & 200.0 & $\mathrm{GPa}$ \\
\hline \% Elongation & 5.8 & & 6.0 & & $1.8 \mathrm{c}$ & \\
\hline Poisson's Ratio & 0.33 & & 0.33 & & 0.25 & \\
\hline
\end{tabular}

a data from the current study

$b$ data from [8]

$\mathbf{c}_{\text {data from manufacturer }}$ 
Table 2. Crack growth rates calculated for various crack locations in titanium hybrid laminates.

$\begin{array}{ccccc}\begin{array}{c}\text { Panel } \\ \text { ID }\end{array} & \begin{array}{c}\text { Applied Stress } \\ \text { MPa }\end{array} & \begin{array}{c}\text { Temperature } \\ { }^{\circ} \mathrm{C}\end{array} & \text { Location of Crack } & \begin{array}{c}\text { Crack Growth Rate } \\ \text { mm/cycle }\end{array} \\ 1 & 130 & 25 & \text { Front-Left } & 1.67 \mathrm{E}-5 \\ 1 & 130 & 25 & \text { Back-Right } & 1.37 \mathrm{E}-5 \\ 2 & 211 & 25 & \text { Back-Right } & 6.84 \mathrm{E}-5 \\ 2 & 211 & 25 & \text { Front-Right } & 9.80 \mathrm{E}-5 \\ 2 & 211 & 25 & \text { Front-Left } & 4.30 \mathrm{E}-5 \\ 2 & 211 & 25 & \text { Back-Left } & 6.01 \mathrm{E}-5 \\ 2 & 208 & 177 & \text { Front-Left } & 8.25 \mathrm{E}-5 \\ 2 & 208 & 177 & \text { Front-Right } & 1.60 \mathrm{E}-4\end{array}$

a Location assumes viewing from the front. 

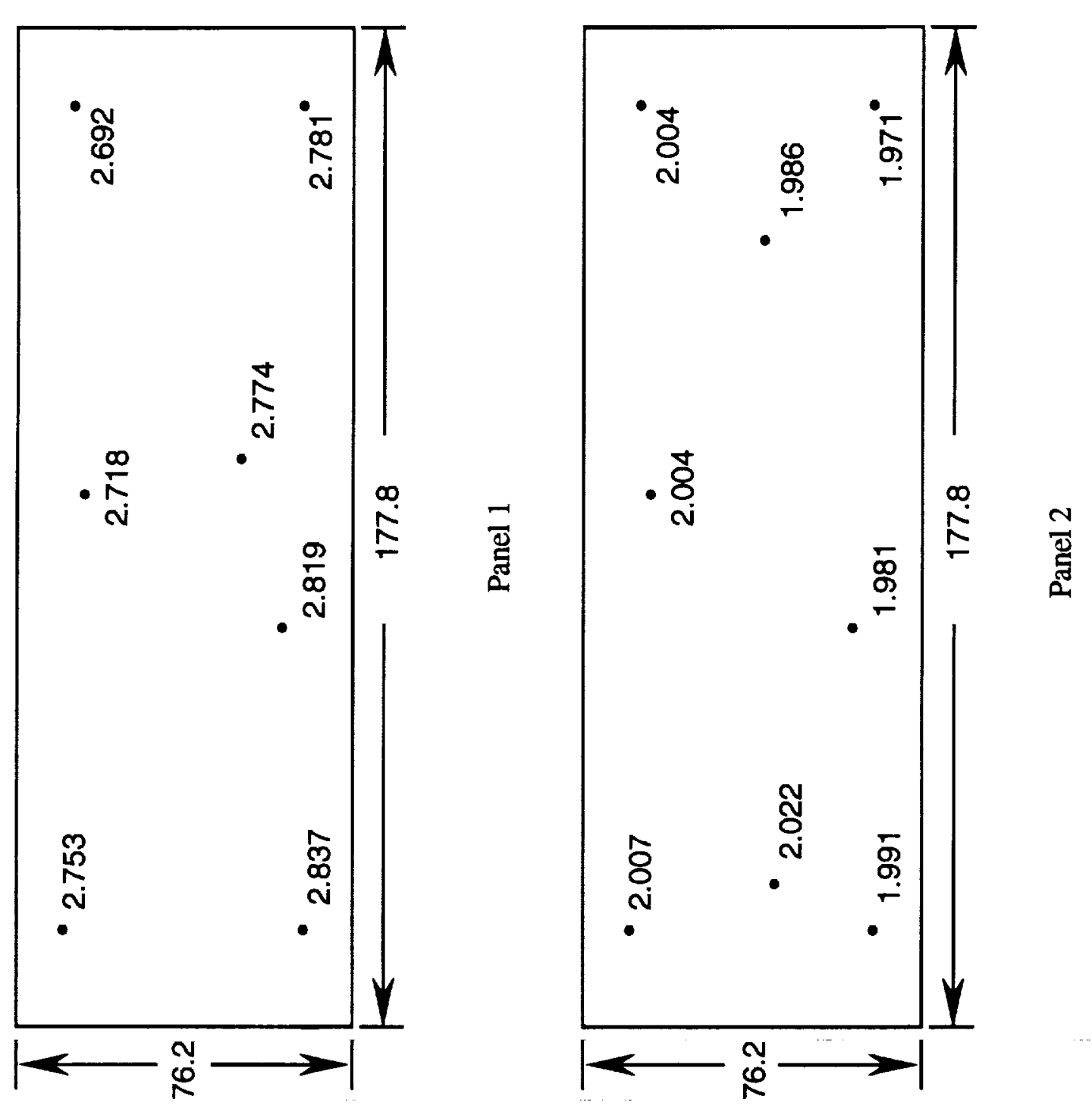

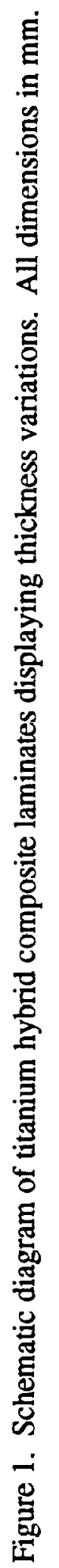




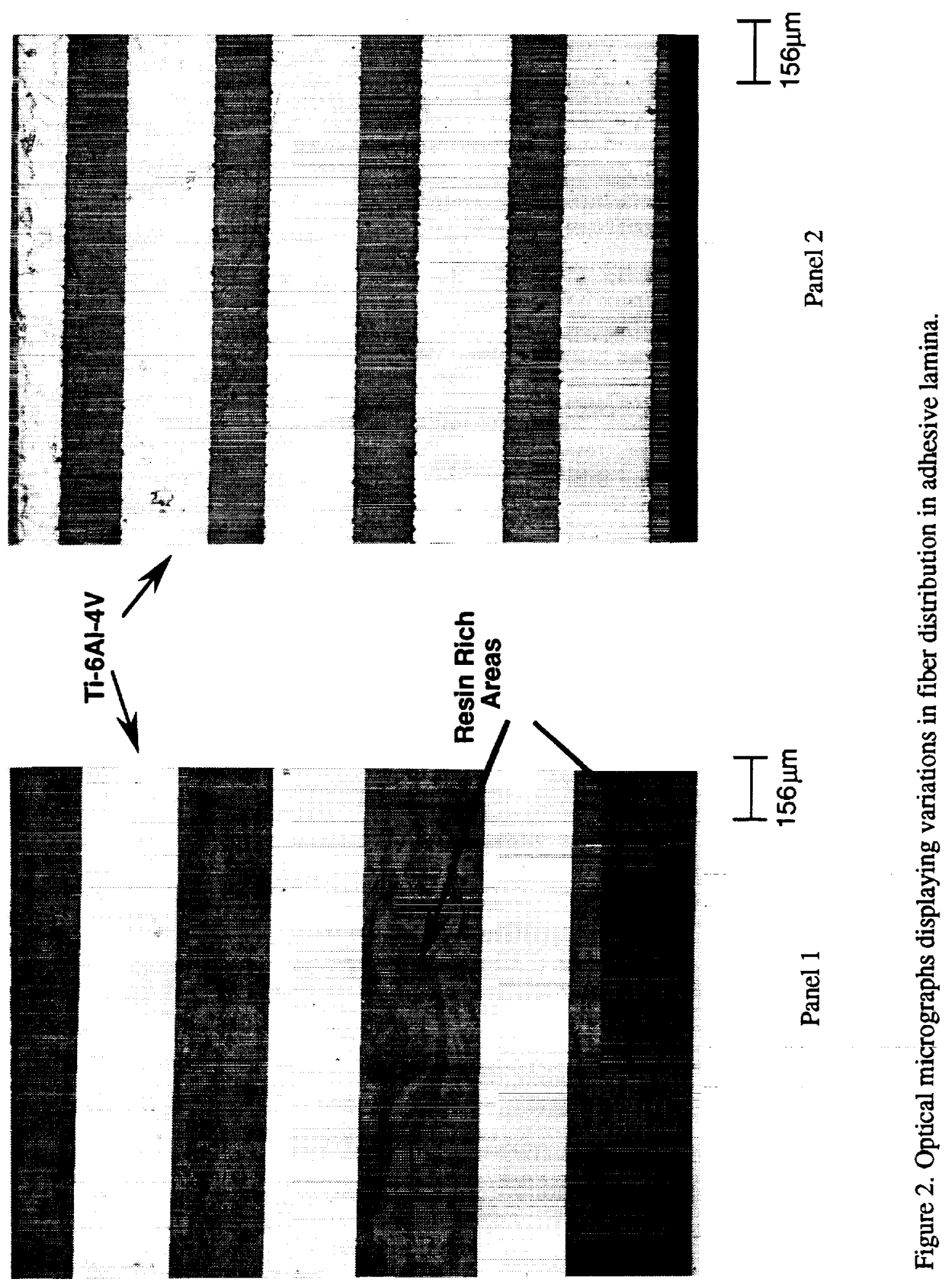




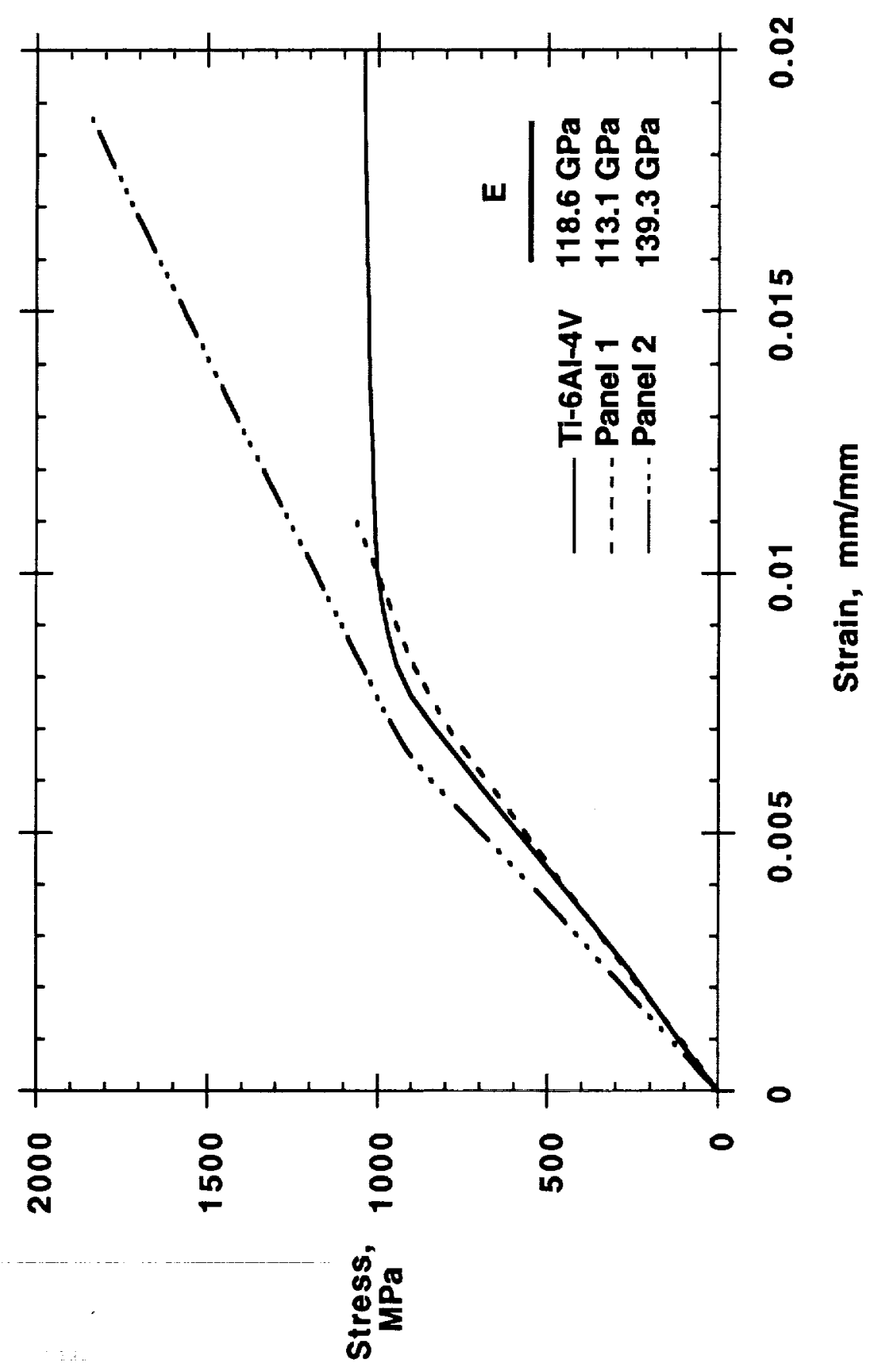

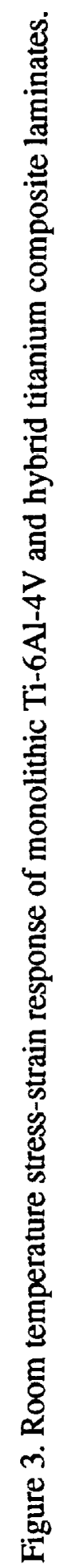



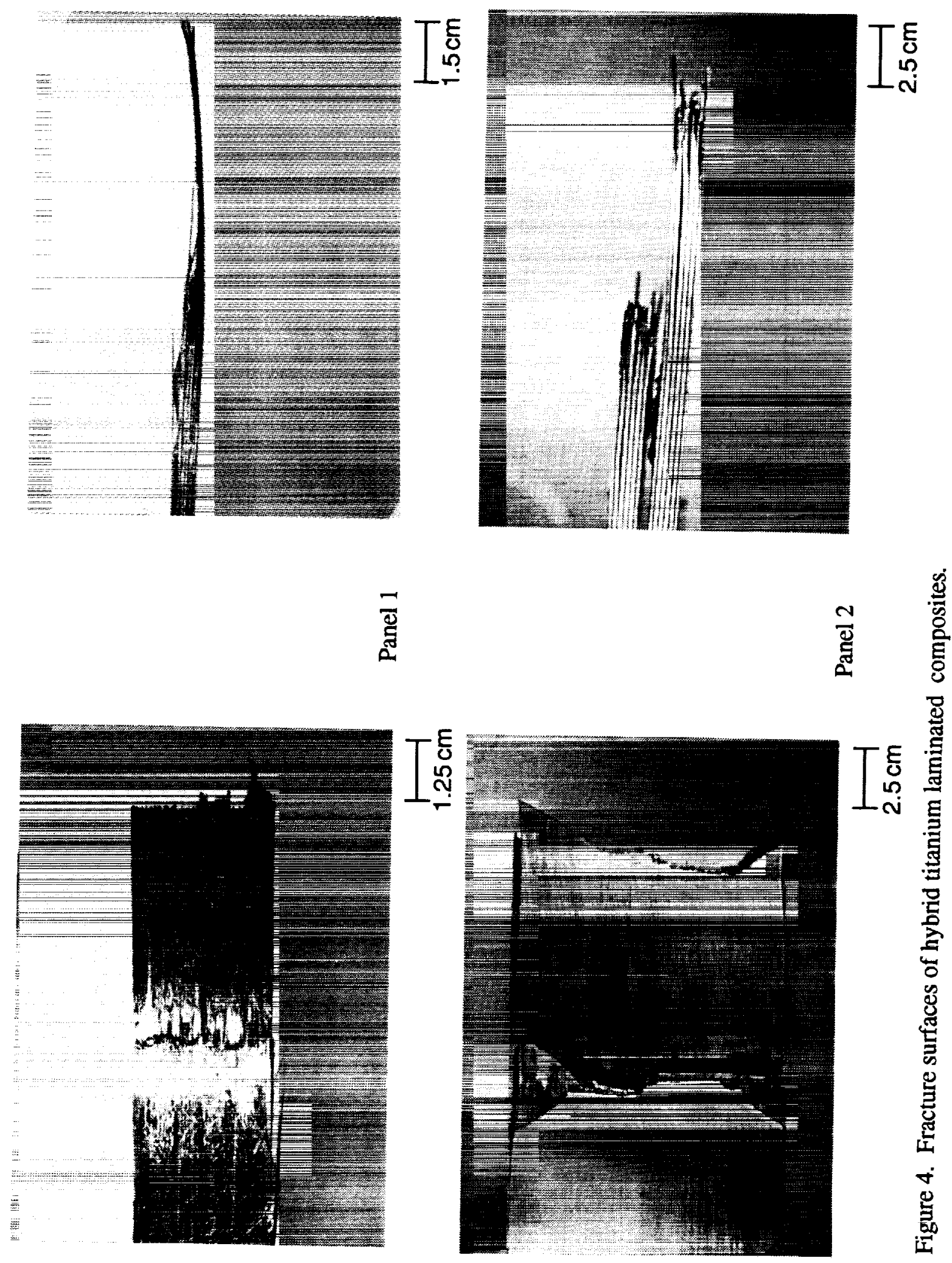

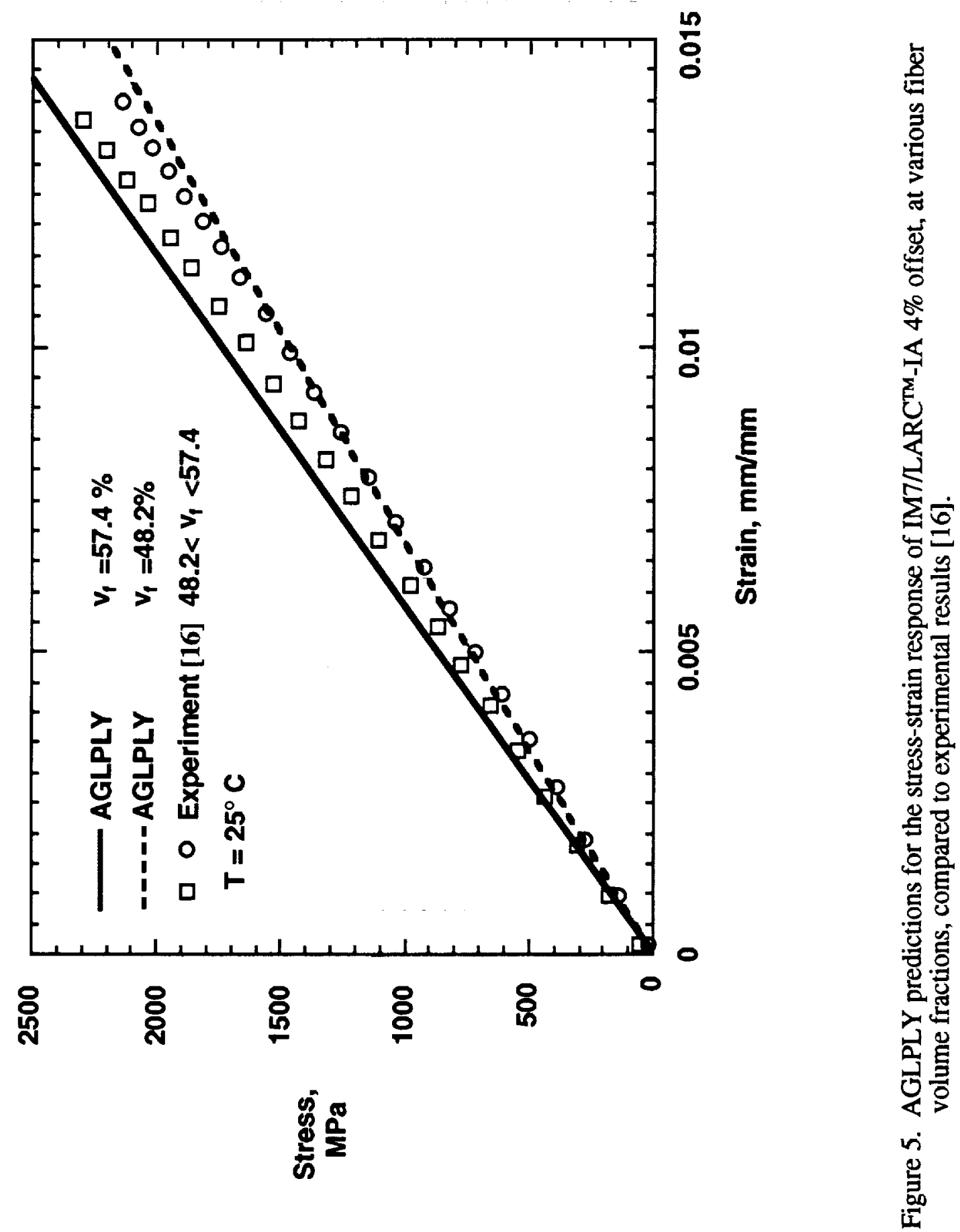

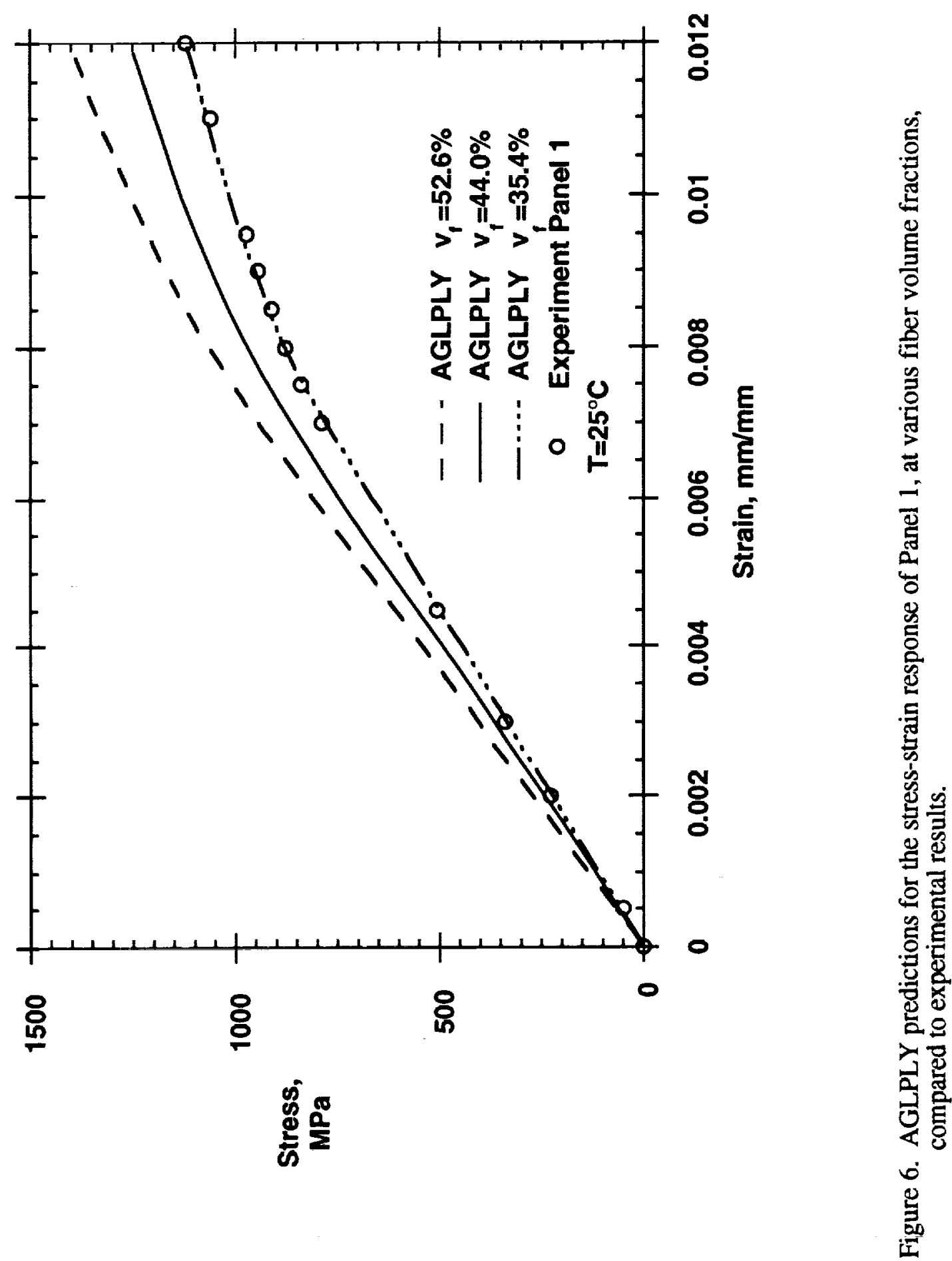

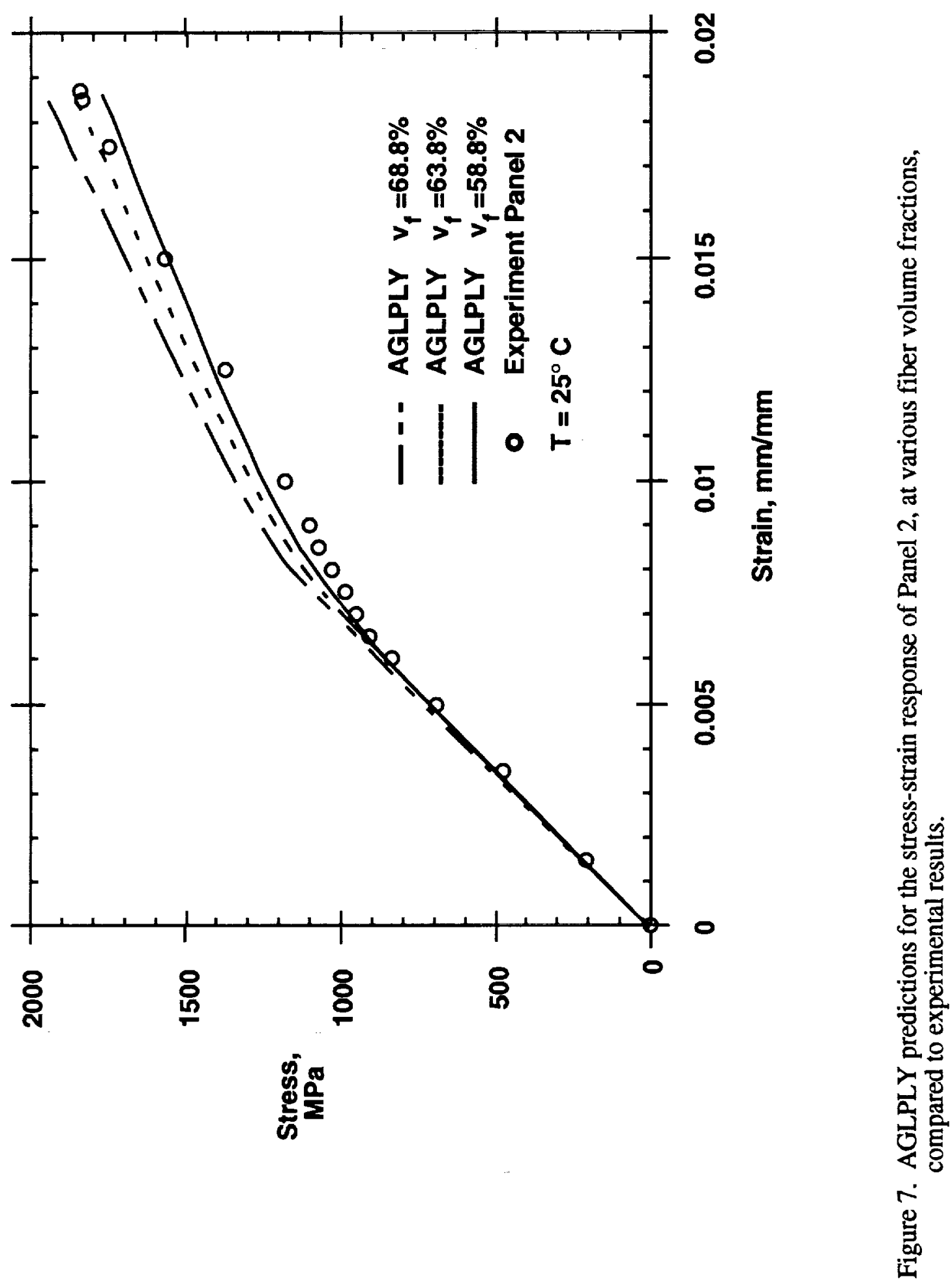

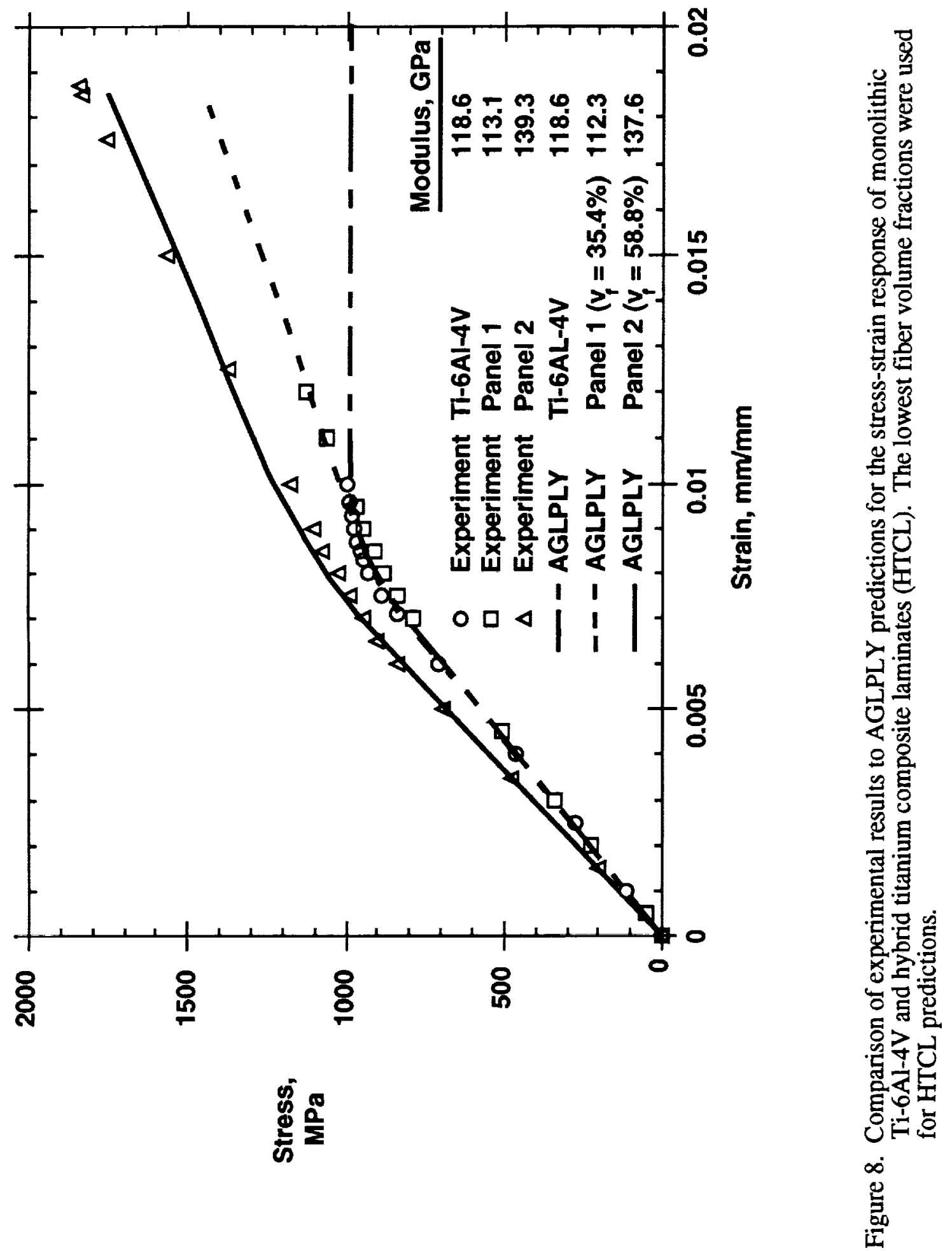


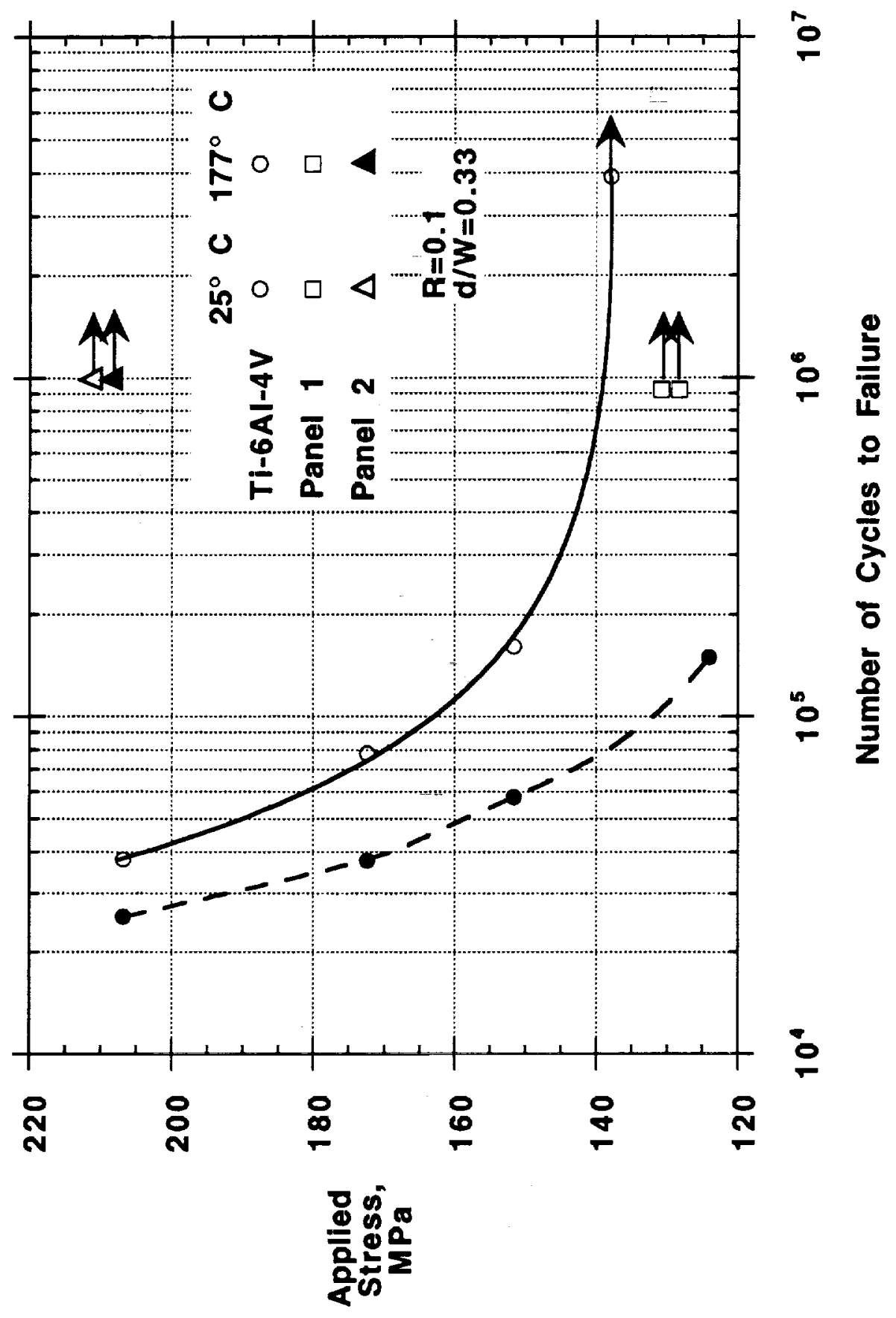

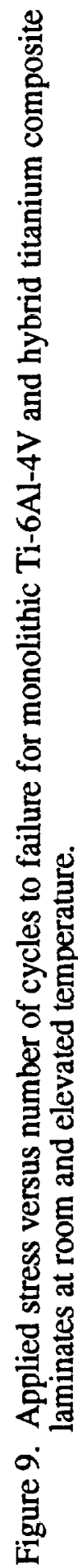




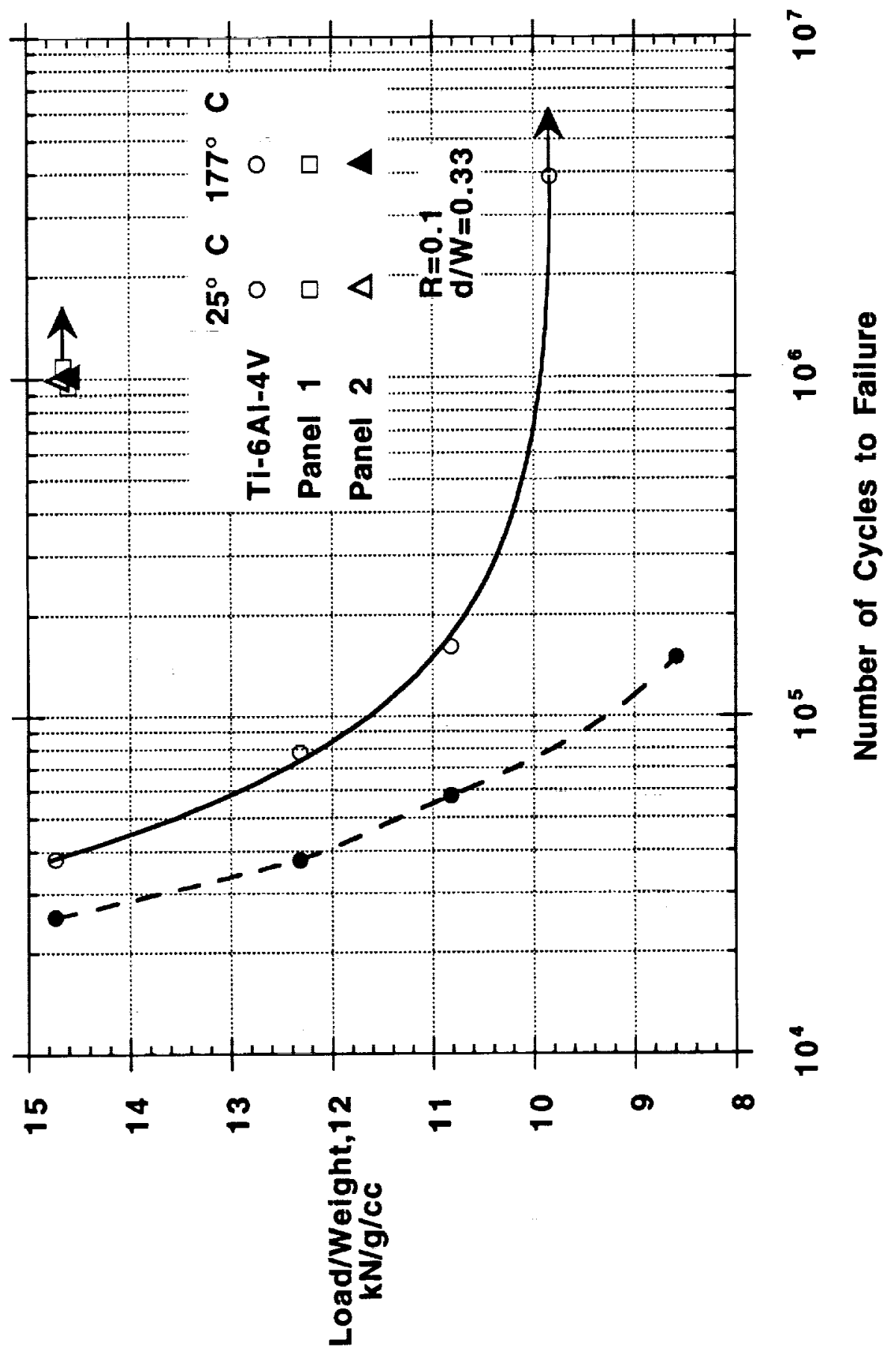

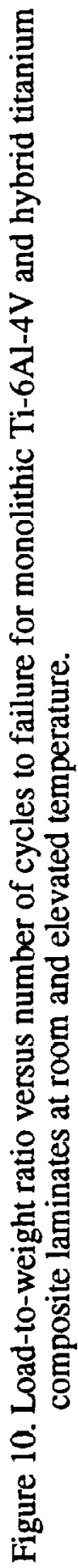




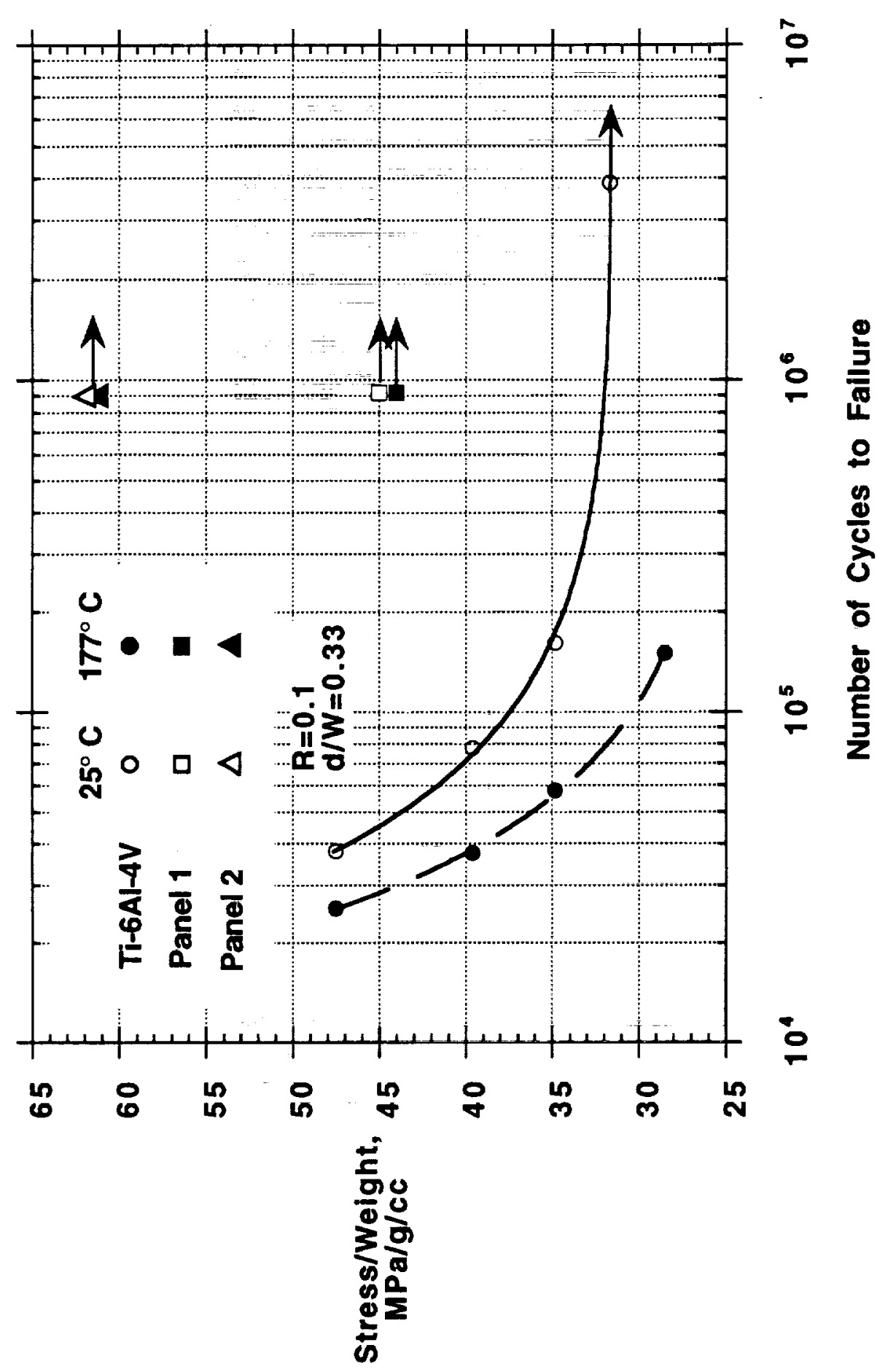

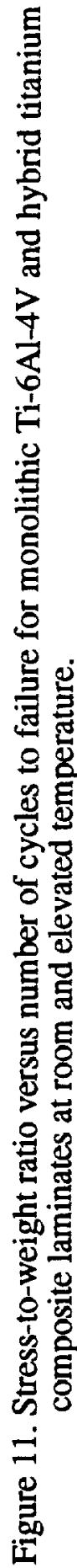




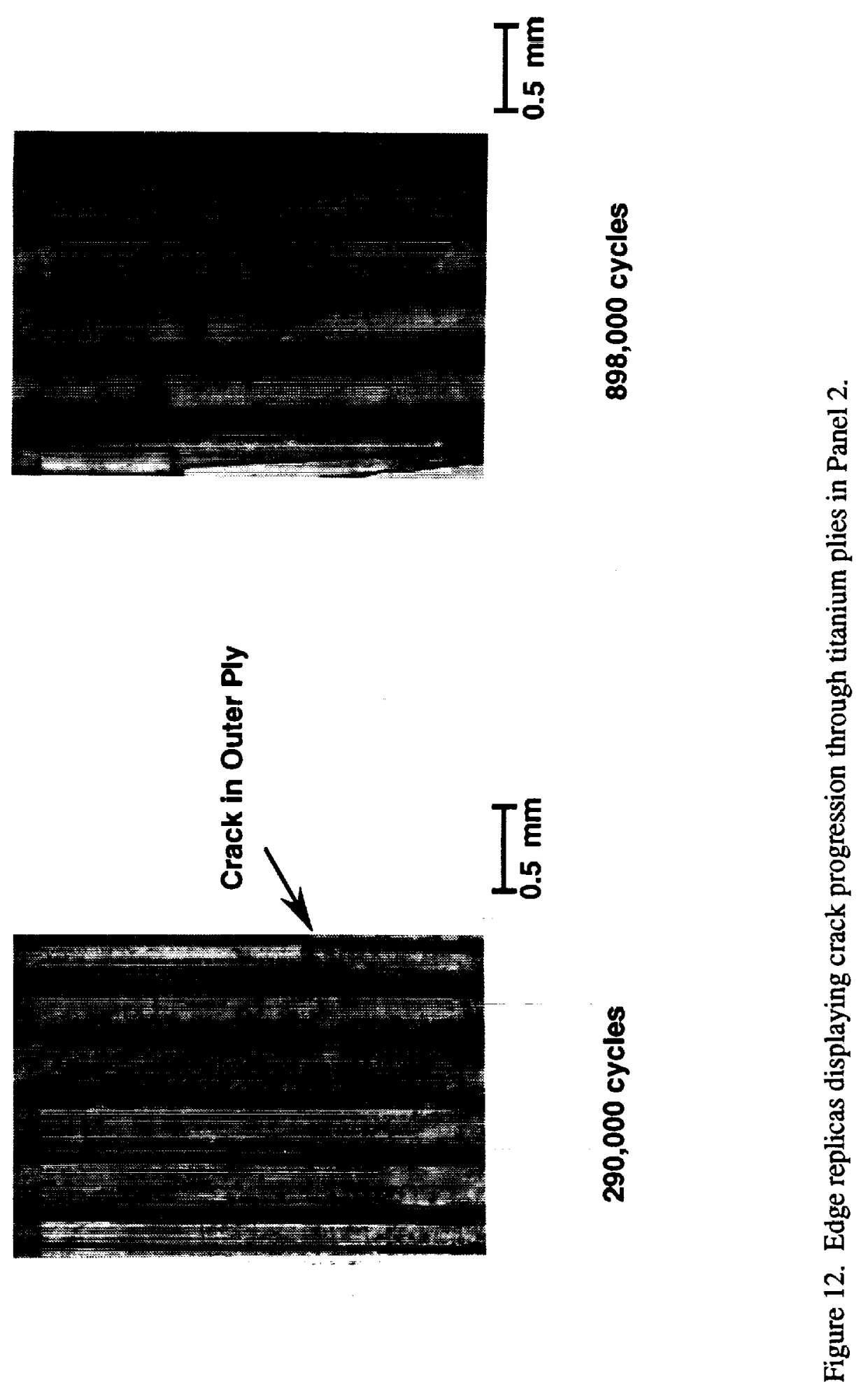




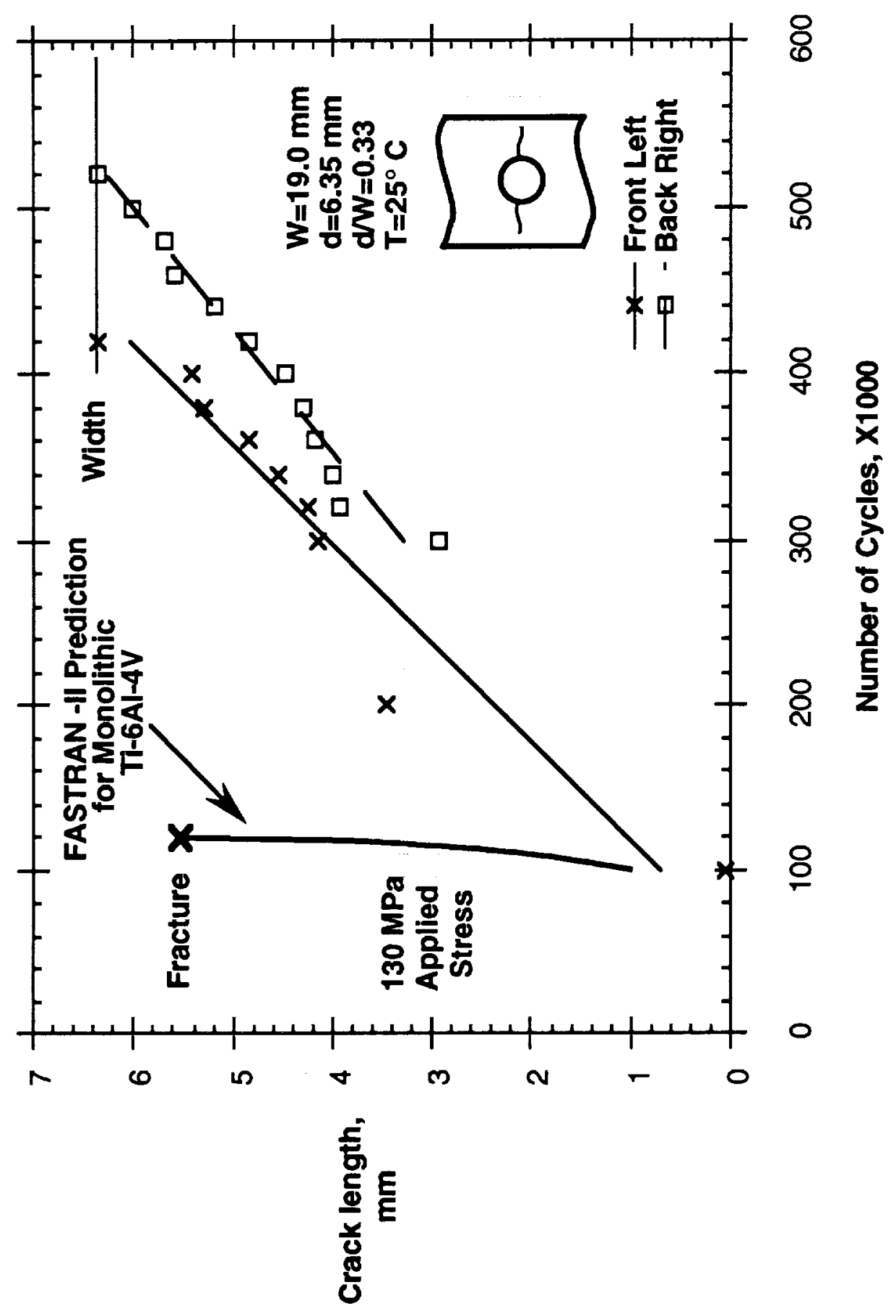

돓

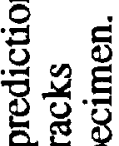

西

它导

$\$$ 舫

응

政

옹용

D

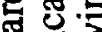

을. 홍

8 过

造的

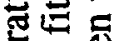

$\$$

害骂

를

8 웅

홍 공

8 过

क्ष

900

히는 등

तै

흐응

至是

\&

군

ภ녕

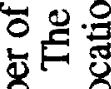

突守高

준을

获

ए

응

305

헝ㅎㅇ 원

45

路客

论응

m

닉

in 

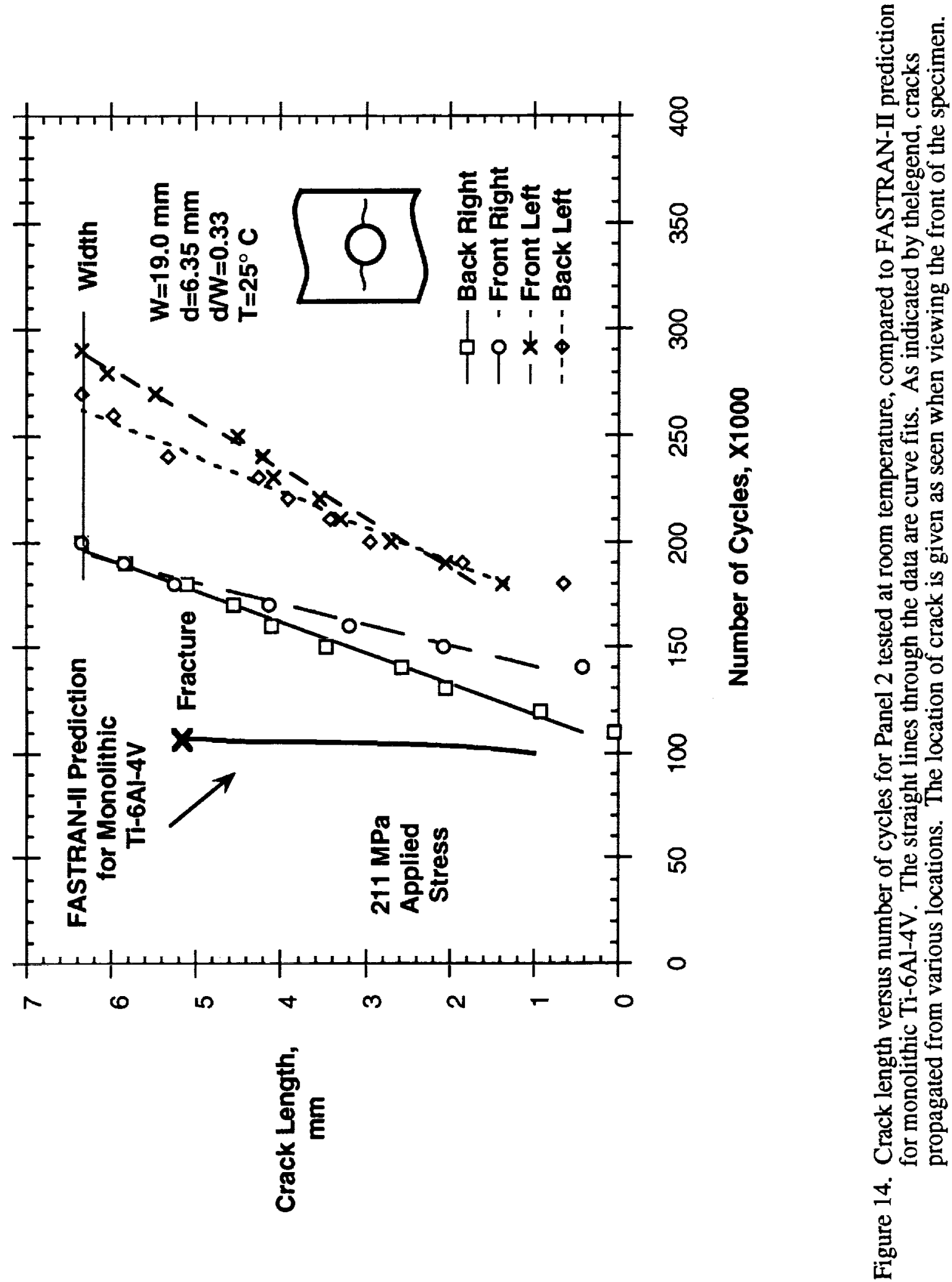

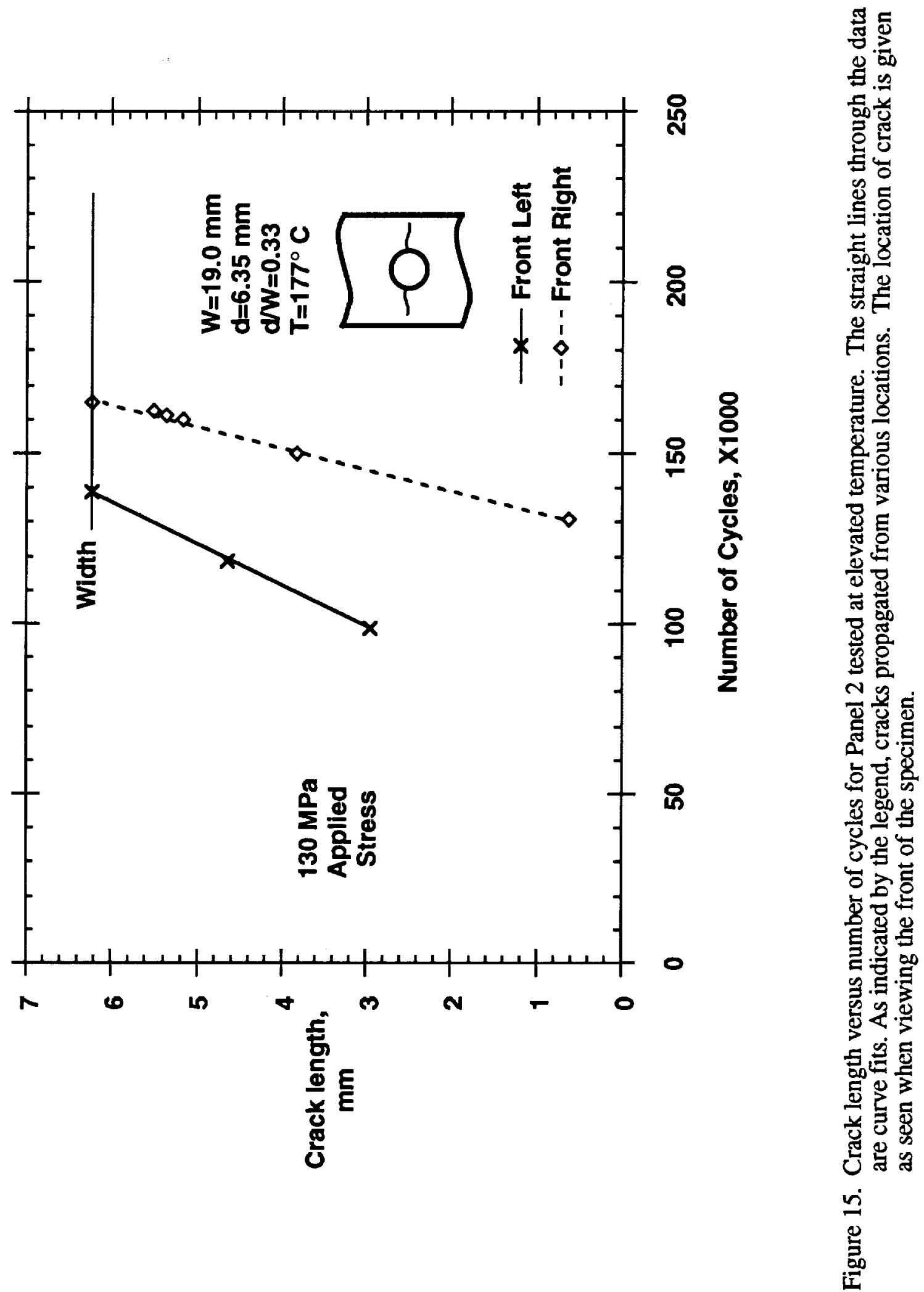


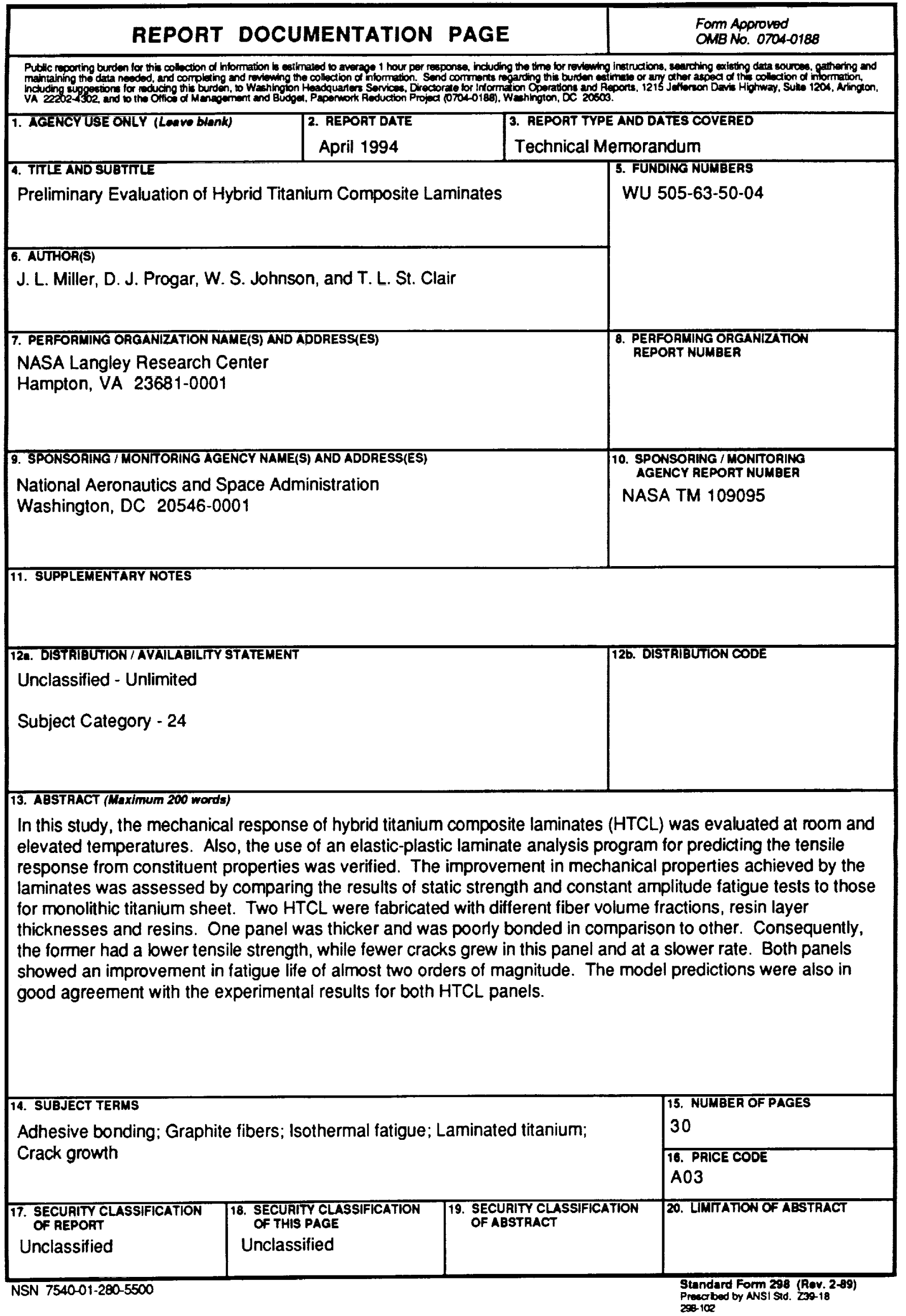

